\title{
Adaptive Control for Focusing of Optical Drive Read/Write Heads
}

\author{
Tsu-Chin Tsao and Steve Gibson \\ Mechanical and Aerospace Engineering \\ University of California, Los Angeles 90095-1597 \\ K-C. Chiu and Shean-Jen Chen \\ Department of Engineering Science \\ National Cheng Kung University \\ Tainan, TAIWAN
}

\begin{abstract}
This paper presents an adaptive control scheme and experimental results for the focusing of an optical readwrite head for optical data storage drives. The adaptive control applies recursive least squares (RLS) algorithm to predict output disturbance and dynamically computes control commands to minimize the output focusing error. The adaptive control could become unstable when significant modeling errors and uncertainties exist. An extension is made to the existent adaptive control scheme to achieve robust stability while sacrificing minimally the control performance. Simulation and experiment conducted on the optical focusing of a digital video recording drive's read-write head are presented to demonstrate the effects of the proposed adaptive control scheme on stability and performance.

Index Terms - optical disk drive, adaptive control, autofocusing.
\end{abstract}

\section{INTRODUCTION}

$\mathbf{I}$ $\mathrm{N}$ the fields of optical storage and optical imaging systems, many applications require precise focusing of optical waves or laser beams subjected to vibration of optical housing and components that disturbs the focusing quality. Vibration-induced jitter typically consists of multiple narrow and broad bandwidths produced by such excitations as device rotating motion, manipulation, and transportation compounded with vibration modes of the structure supporting the optical system. In this paper, we consider focusing control using a digital video recording read/write head (pickup head), which has lightly damped elastic modes [1], [4][8], [13], [25], [27], [28]. This complex combination of disturbances, which often change with time, necessitates the use of adaptive controllers capable of rejecting disturbances with broadband and varying spectra.

The well known waterbed constraint described by the Bode Integral Sensitivity theorem [26] makes it impossible to achieve the needed broadband jitter rejection with linear time-invariant (LTI) controllers. Recent literature on control of jitter in laser beams has introduced adaptive control methods that reject jitter over much greater bandwidths than those

This work was supported by the U.S Naval Office of Research under Grant N00014 07-1-1063 and the National Science Foundation under Grant CMMI-0751261. achieved by LTI feedback control. For laser beams, adaptive controllers based on least-mean-squares (LMS) adaptive filters are presented in [9], [10], [18], [19], and adaptive controllers based on recursive least-squares (RLS) filters are reported in [20]-[22]. Adaptive control has been used for disturbance rejection in other applications, including the reduction of the read-write head position error in computer disk drives [2], [3], [11], [12], [15], [16], [24]. While LMS algorithms are simple and computationally economical, the more complex RLS algorithms achieve faster convergence and exact minimum-variance steady-state performance.

The hardware and geometry of the pick-up head dictate saturation limits on the magnitude of the control commands. Any fast steering mirrors, but especially the micro mirrors used here and in various optical communications systems, can be damaged if the control commands are too large. Also, because small angles of steering-mirror deflection can produce large beam displacements at remote receivers and targets, the mirror rotation must be limited so that the laser beam hits all reflecting surfaces and sensors in the optical path. Either type of constraint can be binding in field applications.

A common characteristic of minimum-variance controllers, adaptive or not, is that they amplify low-level highfrequency noise while minimizing the mean-square values of the output errors. This results from the fact that such controllers must have large high-frequency gains to predict broadband disturbances. In some applications, this amplification results in control command saturation and generate serious spiking phenomenon in output error produced by the combination of control-signal saturation and amplified highfrequency noise. [23] presents a method for incorporating frequency weighting in the adaptive control loop to constrain the high-frequency gain of the adaptive filter that generates the adaptive control command. The frequency responses of both experimental and theoretically computed steady-state filters demonstrate that the frequency weighting reduces the high-frequency gains. Experimental results show that the frequency weighting indeed reduces the amplification of high-frequency noise and eliminates the spikes in the output 


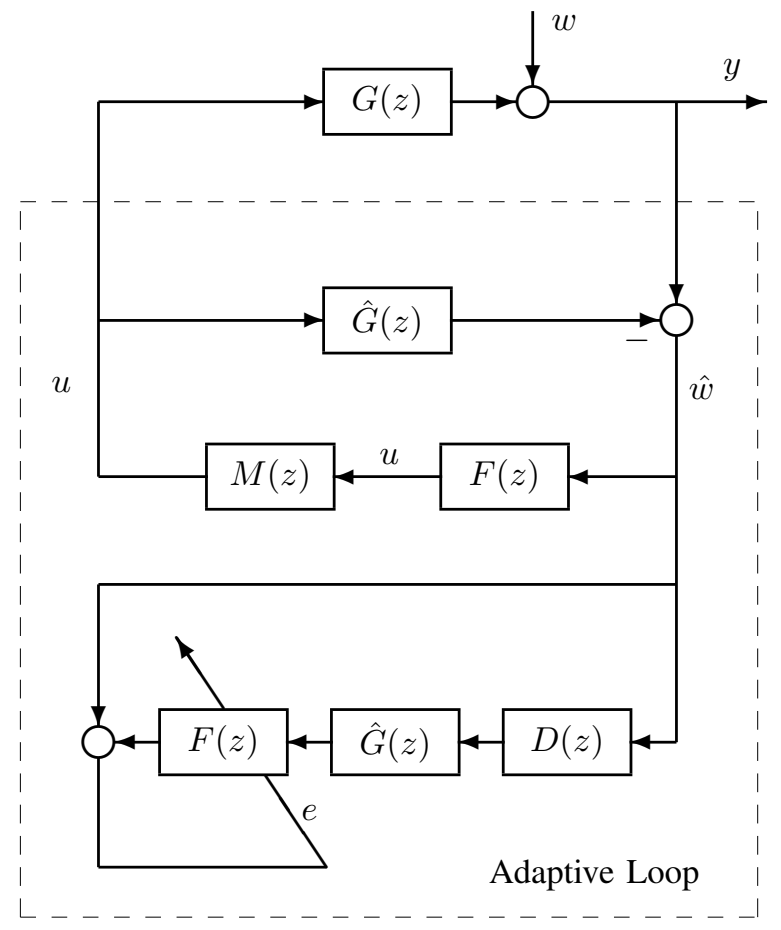

Fig. 1. Block diagram of the adaptive control loop.

error.

In the aforementioned works the plant models closely agree with the actual plant dynamics. In some applications, such as the one considered in this paper, the modeling errors between the plant model and the actual plant dynamics, due to the pick up head flexible structure and sensor nonlinearity, can be substantial and have significant effect on the adaptive control performance or even stability. In this particular case, it is also desirable to constrain the high frequency gains of the adaptive filter, but the aforementioned soft and indirect constraining by frequency weighting in the adaptive filtering is insufficient to render satisfactory results. In this paper we extend our previous adaptive control works by introducing filters in both the feedback control loop and the adaptive filtering loop respectively to explicitly reduce the adaptive controller's high frequency gains and strike the balance of stability and control performance.

The rest of the paper is organized as follows. Section 2 presents the adaptive control scheme with the proposed extension. Section 3 gives the description of the experimental system, followed by the identified plant model in Section 4. The simulation and experimental results are presented in Section 5, followed by the conclusion in Section 6 .

\section{Gain Constrained Adaptive Control}

The class of minimum-variance adaptive controllers considered in this paper is illustrated in Fig. 1. All signals are considered scalar sequences and all blocks are SISO systems. The transfer functions $G(z)$ and $\hat{G}(z)$ are the true plant and the plant model, respectively, both assumed to be stable. This control structure is closed-loop stable if $G(z)=\hat{G}(z)$ and
$F(z)$ is stable. In the adaptive filtering loop, the gains in $F(z)$ are updated to minimize the RMS value of the tuning signal $e$. A copy of $F(z)$ uses the same gains to generate the adaptive control signal $u$.

The adaptive filter $F(z)$ has finite impulse response (FIR), with the lattice realization in [14]. For high orders of $F$, the RLS lattice filter is more computationally efficient and numerically stable than the classical RLS algorithm, which has been used for low-order filters in various adaptive-control applications (e.g., [11], [12], [15]). As discussed in [14], [20], [21], the order-recursive structure of the lattice filter allows variable-order adaptive control. During adaptation, latticefilter generates adaptive control commands of all orders $n \leq L$, with the order increasing to the maximum order $L$ in steady-state. For the experiments and simulations in this paper, values of $L$ up to 256 were used.

The blocks $M$ and $D$ are used to improve the robustness of the adaptive controller with respect to plant modeling error $G-\hat{G}$, which is largest at high frequencies. A sufficient condition for input-output stability of the adaptive system is

$$
\|(G-\hat{G}) M F\|_{\infty}<1 .
$$

This implies that the norm of the gain of the filter $M F$ should be constrained in bandwidths of large modeling error.

The adaptive filter identifies the gains in $F$ to minimize the tuning signal

$$
e=\hat{w}+F \hat{G} D \hat{w}
$$

while the output $y$ satisfies

$$
y=w+G M F \hat{w} .
$$

When (1) holds, $e$ and $y$ satisfy

$$
\begin{aligned}
& e=(1+F \hat{G} D)[1-(G-\hat{G}) M F]^{-1} w, \\
& y=(1+\hat{G} M F)[1-(G-\hat{G}) M F]^{-1} w .
\end{aligned}
$$

With the $R L S$ adaptation, the adaptive filter $F$ becomes slowly time varying and a well known swapping lemma applies to analyze the performance as if $F$ were time invariant, which leads to $F \hat{G} D=\hat{G} D F$. If $G=\hat{G}$ and $D=M$, then $e=y$ in steady state.

The filter $M$ is chosen to be low-pass to limit the highfrequency gain of $M F$. However, if $D=M$, the adaptive filter will identify an $F$ with even larger high-frequency gain than would be case without $M$ because $F$ will attempt to invert the small high-frequency gain of $M$, so that the highfrequency gain of $M F$ is not reduced. Taking $D$ to be allpass with phase delay equal to that of $M$ at all frequencies causes the adaptive filter to identify $F$ with the correct phase, which is the critical issue for closed-loop stability, without causing $F$ to attempt to invert the small high-frequency gain of $M$. This can be done by choosing $M$ to be a linear-phase low-pass filter and $D$ to be the group delay of $M$ (i.e., the time delay that $M$ introduces at all frequencies).

In steady state, it follows from (4) and (5) that

$$
e-y=\hat{G}(D-M) F[1-(G-\hat{G}) M F]^{-1} w .
$$


Hence the tuning signal $e$ and the output error $y$ in steady state differ only in the high-frequency range where the gains of $D$ and $M$ differ.

In this paper, the $M$ and $D$ pairs have the form

$$
M(z)=\left(\frac{z^{2}+2 z+1}{4 z^{2}}\right)^{m}, \quad D(z)=\left(\frac{1}{z}\right)^{m} \text {. }
$$

Filters $M$ of this form have zero gain at the Nyquist frequency for any $m$. The bandwidth of $M$ decreases as $m$ increases, thereby increasing robustness with respect to modeling error in view of (1). However, for larger values of $m, M$ introduces more delay and hence greater disturbance prediction error in the adaptive loop. In subsequent sections, the performance of the adaptive control loop is compared for $m=1$ and $m=2$.

\section{DESCRIPTION OF THE EXPERIMENT}

The experimental setup is based on a DVD pick-up head with wavelength $650 \mathrm{~nm}$, spot diameter $0.659 \mu \mathrm{m}$, focusing depth $0.90 \mu \mathrm{m}$, and linear range $6 \mu \mathrm{m}$. The basic mechanisms of the commercial DVD pick-up head shows in Fig. 2. The DVD pick-up head uses a laser diode to generate light source. The laser beam passes through a diffraction grating, a linear polarizer, a polarized beam splitter, a quarter-wave plate, a collimator and objective lens and focus on the disc. The beam reflected from the disc passes through a quarter wave plate, a polarized beam splitter and a cylindrical lens and projects onto the four-element photodiode array (quadrant detector, QD), which outputs a read-out signal corresponding to the focusing error signal (FES) [7], [17]. When the object is located at correct distance from the focal plane, a circular intensity distribution the photodiode array results. When the object is too far from or too close to the focal plane, an elliptical intensity distribution results. As the disk or objective lens is moved up and down, the changing intensity distribution causes the FES vary in the form of the S-curve shown in Fig. 4. The FES is given by

$$
F E S=\left(V_{a}+V_{c}\right)-\left(V_{b}+V_{d}\right)
$$

where $V_{a}, V_{b}, V_{c}$ and $V_{d}$ represent the output of QD.

Another output is the sum of the intensities on the quadrant detector $\mathrm{QD}$, which is referred to as the RF signal. The RF signal can be used to check the laser spot focus on the disc. The circuit board includes an automatic power control (APC) driver for the laser diode, an operational circuit for the $\mathrm{QD}$, and two motor drivers for the voice coil motor (VCM) and spindle motor. The xPC target machine sends actuator commands to VCM and spindle motor and acquires FES and RF from QD. The sample-and-hold rate for the control system is $2 \mathrm{kHz}$.

\section{PLANT MODELS AND PID CONTROL}

\section{A. The open-loop plant}

The open-loop discrete-time transfer function $G_{O P}(z)$ maps the VCM control command to the sampled output FES. For control design, it is assumed that all signals are sufficiently small that the FES remains in the linear range

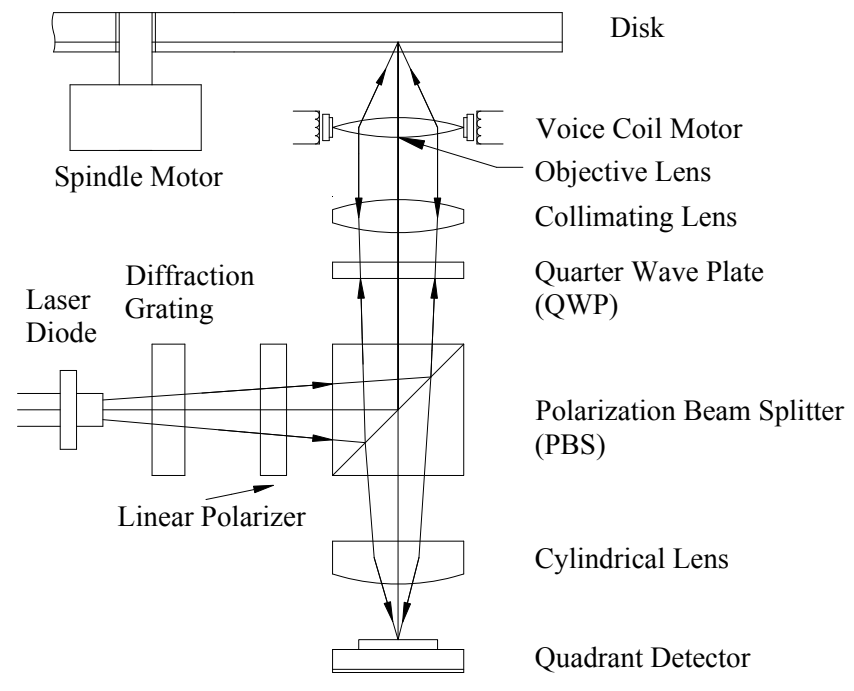

Fig. 2. Structure of the DVD pick-up head.

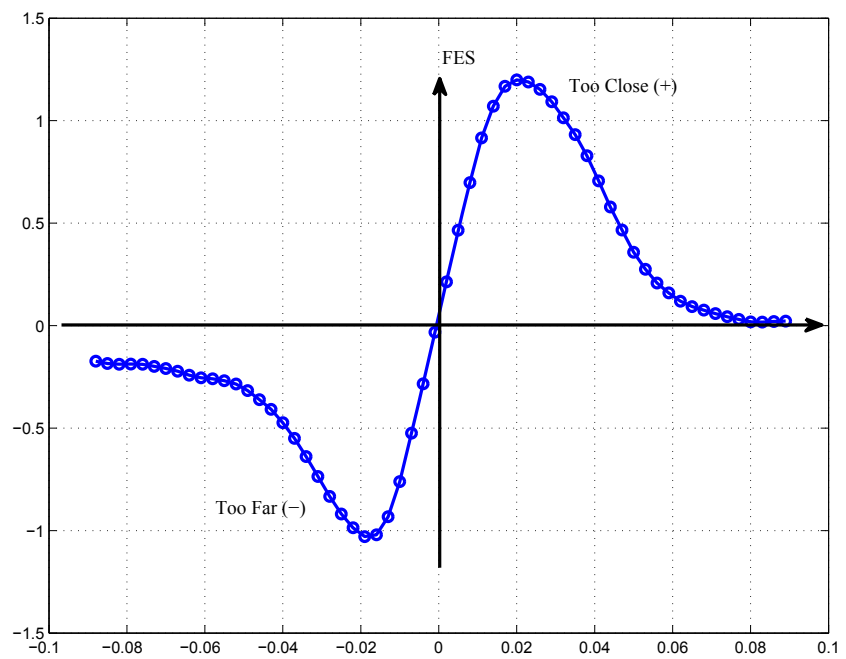

Fig. 4. Focusing error signal vs. distance between the optical pick up head and media surface.

of the $\mathrm{S}$ curve in Fig. 4. The subspace system identification algorithm N4SID in MATLAB was used to identify the linear model

$$
\hat{G}_{O P}(z)=\frac{-1.642 z+2.718}{z^{2}-1.947 z+0.9679}
$$

of the open-loop transfer function from 70,000 samples of input-output data. Fig. 5 shows the bode plot of $\hat{G}_{O P}(z)$.

\section{B. Closed-loop system with PID feedback control}

Prior to adaptive control, a linear time-invariant control loop containing the tuned PID controller

$$
C_{P I D}(z)=\frac{0.24158\left(z^{2}-1.95 z+0.9651\right)}{z(z-1)}
$$

is closed around the open-loop plant. With this loop closed, $N 4 S I D$ was used to identify the closed-loop plant model denoted by $\hat{G}$. 


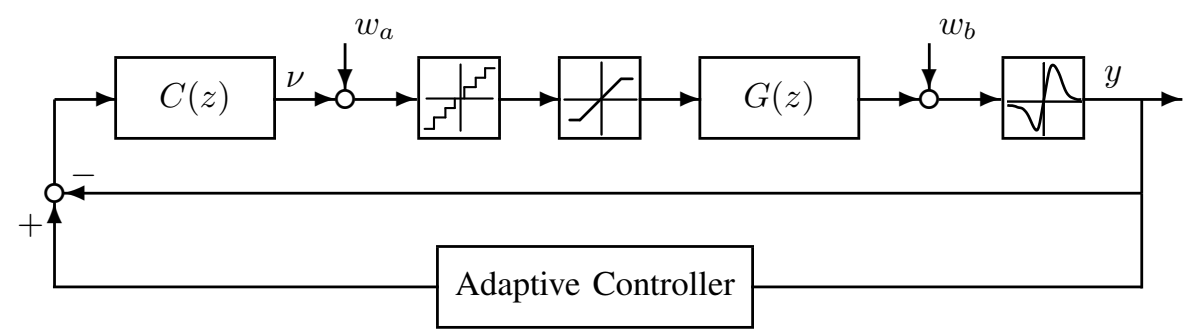

Fig. 3. Block diagram for adaptive control in simulation.

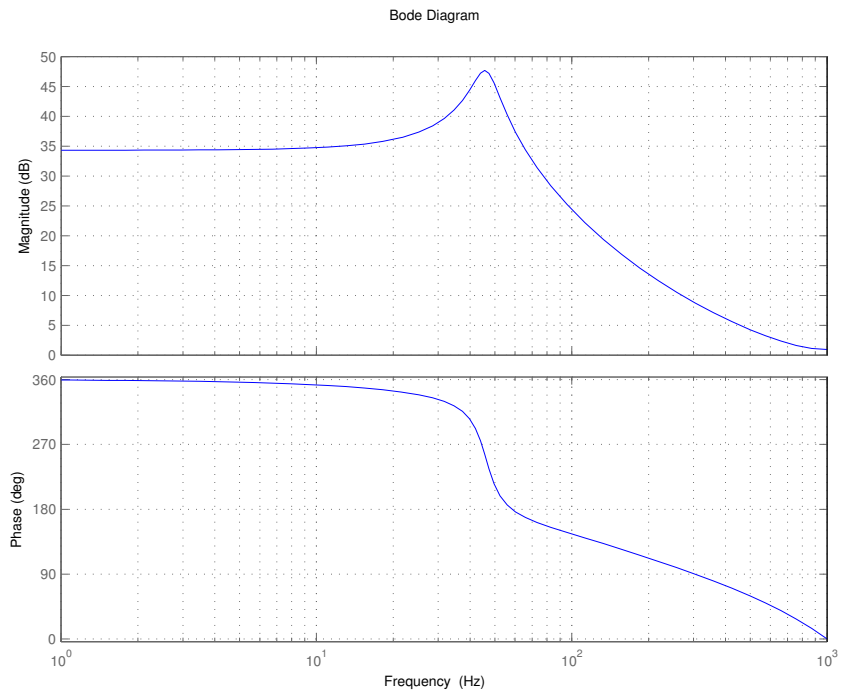

Fig. 5. Bode plots for identified open-loop plant.

With the estimated closed-loop transfer function $\hat{G}$, the estimated closed-loop sensitivity transfer function

$$
\hat{S}=(I+\hat{G} C)^{-1}
$$

is computed. This transfer function, which is shown in Fig. 6, shows that the error-rejection bandwidth is about $80 \mathrm{~Hz}$.

\section{SIMULATION AND EXPERIMENTAL RESULTS}

\section{A. Simulation}

The simulation results were generated for the model in Fig. 3. This model of the DVD and the contorl loops includes a quantizer, a saturation block and the S-curve nonlinearity, all of which are in the physical system. The simulation uses multiple sinusoidal disturbances in the disturbance $w_{a}$ to simulate harmonic disturbances generated by a rotating disc, which are the main disturbances in portable DVD video recorders. In the simulation, the output disturbance $w_{b}$ is taken to be zero. The frequencies and respective amplitudes in the disturbance $w_{a}$ are

$$
\begin{array}{llllll}
5.5 & 11 & 50 & 65 & 75 & (\text { Hz) } \\
0.010 & 0.005 & 0.005 & 0.003 & 0.015 & \text { (volt) }
\end{array} .
$$

Fig. 7 shows time series for the focusing error (FES). Initially only the PID loop is closed, and the adaptive control loop closes at $1.5 \mathrm{sec}$. For the top plot in Fig. 7, the simplest version of the adaptive controller is used, with FIR order 2

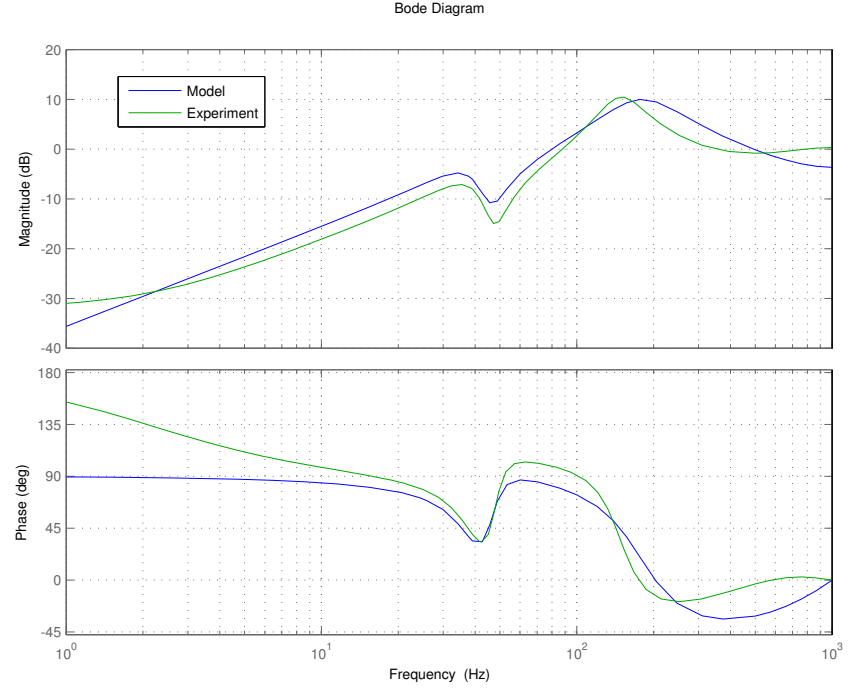

Fig. 6. Bode plots for estimated sensitivity transfer function $\hat{S}$.

and no $M$ filter. For the bottom plot, FIR order is $n=256$ and the $M$ filter has the form in (7) with $m=2$ (denoted by $M^{2}$ ). Fig. 8 shows the power spectral densities of the of the FES signals in Fig. 7, comparing performance with PID control only and with both versions of the adaptive controller.

Table I shows the RMS values of FES for various orders of lattice filter, with and without the $M$ filter and $M^{2}$. The closed-loop system with adaptive control without the $M$ and $D$ filtering is stable only for adaptive filter order $n=2$. That the closed-loop system is unstable for $n>2$ is indicated by $\mathrm{X}$ in Tables $\mathrm{I}$ and II. On the other hand, introduction of the properly designed $M$ filter and corresponding delay $D$ renders stable, robust performance for all orders up to $n=256$ (the highest order tried). As expected, the RMS value of the FES decreases with increasing lattice order. The RMS value of the FES with PID control only is 0.4067 .

\section{B. Experiment}

Experiments were performed with the DVD not spinning, but with the multi-frequency sinusoidal disturbance $w_{a}$ introduced by the actuator. Having the same disturbance in both simulation and experiment allowed direct comparison of simulation and experimental results. In the experiment the voice coil motor (VCM) generated both the control commands and the disturbance $w_{a}$, although the disturbance signals were not available to the control loops. The FES signals for PID 

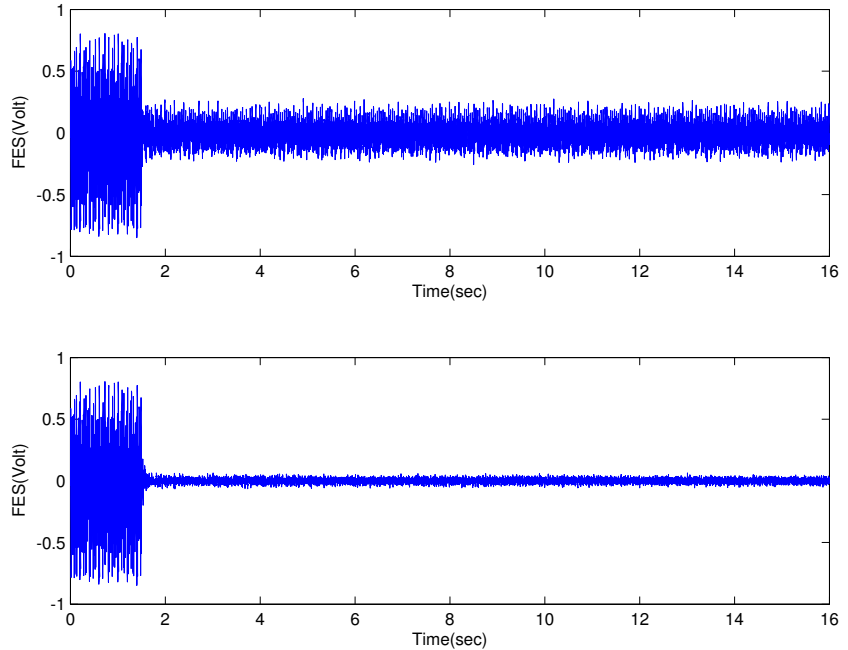

Fig. 7. Simulation results. Top: adaptive control with adaptive filter order $n=2$ with no $M$ filter; bottom: adaptive control with adaptive filter order $n=256$ and $M$ filter with $m=2$.

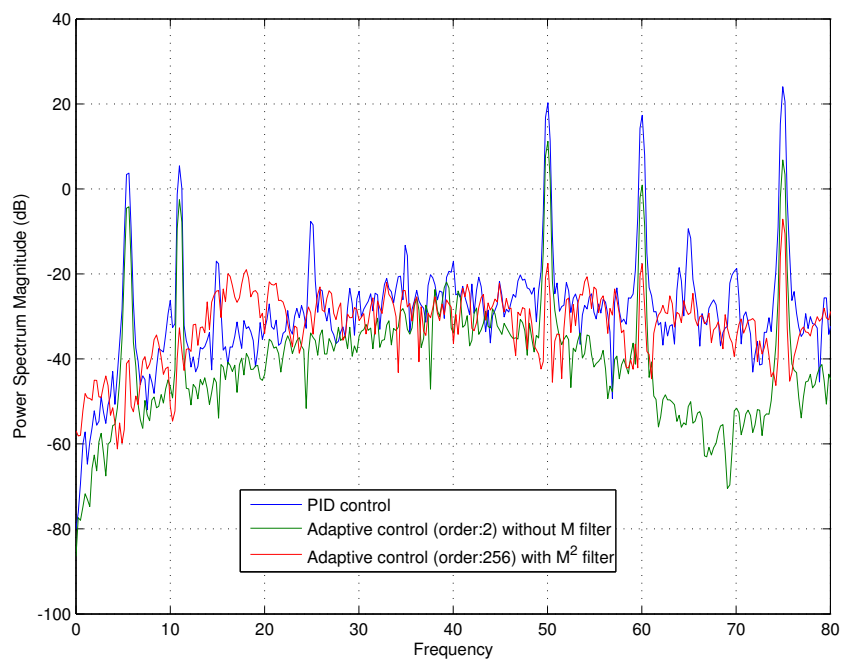

Fig. 8. Simulation results. PSD of FES for PID control only and for adaptive control with adaptive filter order $n=2$ with no $M$ filter and for adaptive control with adaptive filter order $n=256$ and $M$ filter with $m=2$.

control only and several versions of the adaptive controller are compared in Table II. The experimental and simulation results are similar in several respects, including transient and steady-state performance of the adaptive control loop. In particular, the importance of the $M$ filter and delay $D$ is evident and the RMS value of the FES decreases as the order of the adaptive lattice filter increases. Also, in both simulation and experiment, the $M$ filter is necessary for closed-loop stability with adaptive control when the order of the lattice filter is greater than 2 .

\section{CONCLUSIONS}

This paper has introduced a disturbance-rejecting adaptive control scheme with improved robustness to plant modeling error at high frequencies. Simulation results and real-time
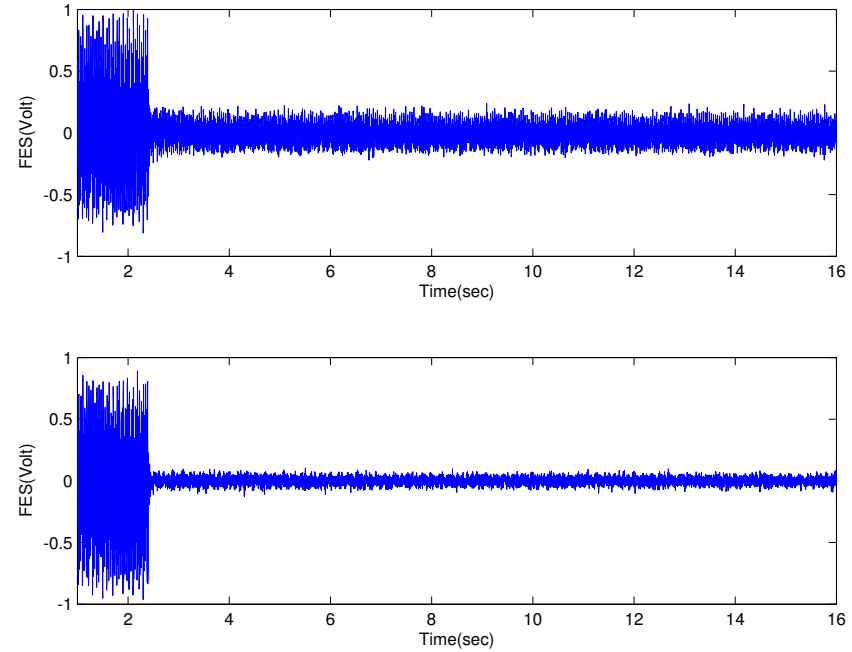

Fig. 9. Experimental results. Top: adaptive control with adaptive filter order $n=2$ with no $M$ filter; bottom: adaptive control with adaptive filter order $n=256$ and $M$ filter with $m=2$.

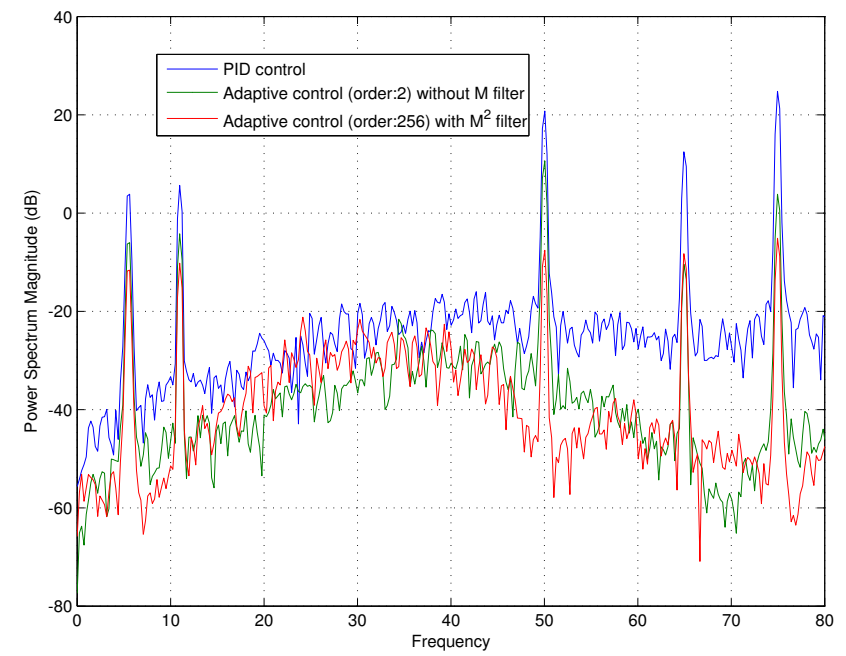

Fig. 10. Experimental results. PSD of FES for PID control only and for adaptive control with adaptive filter order $n=2$ with no $M$ filter and for adaptive control with adaptive filter order $n=256$ and $M$ filter with $m=2$.

implementation on focusing a DVD optical head under dynamic disturbances have demonstrated the necessity and effectiveness of the proposed method for robustness enhancement. Although the steady-state output error produced by the adaptive controller in the paper is larger at high frequencies than what would be produced by a minimum-variance controller designed with no robustness consideration, the simulation and experimental results show large reductions in output error compared to the performance of a well tuned PID controller.

The combination of a low-pass linear-phase filter $M$ in the adaptive control loop and a phase-matching all-pass delay in the tuning loop introduces a frequency-weighting effect that reduces the high-frequency gain of the adaptive controller, thereby increasing robustness with respect to high-frequency 
TABLE I

SiMULATION RESULTS: RMS FES VALUES.

\begin{tabular}{|l|c|c|c|c|c|c|c|c|}
\hline & \multicolumn{8}{|c|}{ Order $n$ of lattice filter $F$} \\
\hline & 2 & 4 & 8 & 16 & 32 & 64 & 128 & 256 \\
\hline Adaptive control without $M$ & 0.0923 & $\mathrm{X}$ & $\mathrm{X}$ & $\mathrm{X}$ & $\mathrm{X}$ & $\mathrm{X}$ & $\mathrm{X}$ & $\mathrm{X}$ \\
\hline Adaptive control with $M$ & 0.1095 & 0.0805 & 0.0772 & 0.0623 & 0.0473 & 0.0360 & 0.0223 & 0.0151 \\
\hline Adaptive control with $M^{2}$ & 0.1303 & 0.1015 & 0.0964 & 0.0744 & 0.0570 & 0.0440 & 0.0248 & 0.0170 \\
\hline
\end{tabular}

TABLE II

EXPERIMENTAL RESULTS: RMS FES VALUES.

\begin{tabular}{|l|c|c|c|c|c|c|c|c|}
\hline & \multicolumn{9}{|c|}{ Order of Lattice filter } \\
\hline & 2 & 4 & 8 & 16 & 32 & 64 & 128 & 256 \\
\hline Adaptive control & 0.0810 & $\mathrm{X}$ & $\mathrm{X}$ & $\mathrm{X}$ & $\mathrm{X}$ & $\mathrm{X}$ & $\mathrm{X}$ & $\mathrm{X}$ \\
\hline Adaptive control with $M$ & 0.0950 & 0.0478 & 0.0416 & 0.0279 & 0.0233 & 0.0212 & 0.0184 & 0.0172 \\
\hline Adaptive control with $M^{2}$ & 0.1218 & 0.0877 & 0.0537 & 0.0484 & 0.0294 & 0.0285 & 0.0269 & 0.0262 \\
\hline
\end{tabular}

modeling error. Both simulations and the experimental application to the DVD optical head show that, without $M$ the closed-loop system is unstable if the order of the lattice filter in the adaptive controller is greater than 2, whereas with $M$ the closed-loop system is stable for adaptive filter orders up to 256. The larger adaptive filter order yields much smaller output error variance.

\section{REFERENCES}

[1] Jong-Shik Baek, Chung Choo Chung, and Masayoshi Tomizuka, Antishock controller design for optical disk drive systems with a nonlinear controller, American Control Conference (Minneapolis, Minnesota), 2006, pp. 1982-1989.

[2] M. Bodson and S. C. Douglas, Adaptive algorithms for the rejection of sinusoidal disturbances with unknown frequency, Automatica 33 (1997), no. 12, 2213-2221.

[3] M. Bodson, A. Sacks, and P. Khosla, Harmonic generation in adaptive feedforward cancellation schemes, IEEE Transactions on Automatic Control 39 (1994), no. 9, 1939-1944.

[4] C. S. Chang and T. S. Liu, $L Q G$ controller for active vibration absorber in optical disk drive, IEEE Transactions on Magnetics 43 (2007), no. 2, 799-801.

[5] Chi-Shen Chang and Tzong-Shi Liu, Design of adaptive dynamic absorber to reduce optical disk drive vibration at multiple rotating speeds, Japanese Journal of Applied Physics 45 (2006), no. 2B, 11201123.

[6] Yong C. Choi and Chang-Hun Kim, Adaptive fuzzy logic for focusing in optical disk player, IEEE Transactions on Consumer Electronics 54 (2008), no. 2, 545-550.

[7] Donald K. Cohen, Wing Ho Gee, M. Ludeke, and Julian Lewkowicz, Automatic focus control: The astigmatic lens approach, Applied Optics 23 (1984), no. 4, 565-570.

[8] T. Narahara et al., Optical disc system for digital video recording, Japanese Journal of Applied Physics 39 (2000), 912-919.

[9] L. P. Fowler and R. Blankinship, Experimental Adaptive Filtering and Disturbance Feedforward Approach for Flexible Beam Train Control with Single Disturbance Path, in Proc. 2006 DEPS Directed Energy Systems Symposium: Beam Control Conference.

[10] R. M. Glaese, E. H. Anderson, and P. C. Janzen, Active suppression of acoustically induced jitter for the airborne laser, in Proc. SPIE 4034, Laser Weapons Technology.

[11] R. Horowitz and B. Li, Adaptive track-following servos for disk file actuators, IEEE Transactions on Magnetics 32 (1996), no. 3, 17791786.

[12] R. Horowitz, B. Li, and J. W. McCormick, Wiener-filter-based Minimum Variance Self-tuning Regulation, Automatica 34 (1998), no. 5, 531-544.

[13] Seiji Imagawa, Motoyuki Suzuki, Hiroaki Ono, and Jun Ohya, Actuator control to supress disturbances for $8 \mathrm{~cm} d v d$-ram video camera recoder, IEEE Transactions on Consumer Electronics 47 (2001), no. 3, $528-533$.
[14] S.-B. Jiang and J. S. Gibson, An unwindowed multichannel lattice filter with orthogonal channels, IEEE Transactions on Signal Processing $\mathbf{4 3}$ (1995), no. 12, 2831-2842

[15] K. Krishnamoorthy and T.-C. Tsao, Adaptive- $Q$ with LQG stabilizing feedback and real time computation for disk drive servo control, in Proc. of the American Control Conference (Boston, MA, Jun. 2004, pp. 1171-1175).

[16] Kalyanam Krishnamoorthy and Tsu-Chin Tsao, Experimental study of adaptive-q control for disk drive track-following servo probleml, IEEE/ASME Transactions on Mechatronics 15 (2010), no. 3, 480491.

[17] Masud Mansuripur, Analysis of astigmatic focusing and push-pull tracking error signal in magnetooptical disk system, Applied Optics 26 (1987), no. 18, 3981-3986.

[18] M. A. McEver, D. G. Cole, and R. L. Clark, Experiments in adaptive optical jitter control, in Proc. SPIE 5049, Smart Structures and Materials 2003: Modeling, Signal Processing, and Control.

[19] _ Adaptive feedback control of optical jitter using Qparameterization, Optical Engineering 43 (2004), no. 4, 904-910.

[20] P. K. Orzechowski, N. Y. Chen, J. S. Gibson, and T.-C. Tsao, Optimal suppression of laser beam jitter by high-order rls adaptive control, IEEE Transactions on Control Systems Technology 16 (2008), no. 2, 255-267.

[21] N. O. Pérez Arancibia, N. Chen, S. Gibson, and T.-C. Tsao, Variable order adaptive control of a microelectromechanical steering mirror for suppression of laser beam jitter, Optical Engineering 45 (2006), no. $10,104206-1-12$.

[22] _ Adaptive control of a MEMS steering mirror for supression of laser beam jitter, in Proc. of the American Control Conference (Portland, OR, Jun. 2005, pp. 3586-3591).

[23] N. O. Pérez Arancibia, J. S. Gibson, and T.-C. Tsao, Frequencyweighted minimum-variance adaptive control for laser beam jitter suppressionl, IEEE/ASME Transactions on Mechatronics 14 (2009), no. $3,337-348$

[24] A. Sacks, M. Bodson, and P. Khosla, Experimental Results of Adaptive Periodic Disturbance Cancellation in a High Performance Magnetic Disk Drive, ASME Journal of Dynamic Systems, Measurement, and Control 118 (1996), no. 3, 416-424.

[25] H. Sekiya and S. Kagami, Stabilization of focus servo system for highspeed access in a $90 \mathrm{~mm}$ mo disk drive, Japanese Journal of Applied Physics 31 (1992), 648-652.

[26] S. Skogestad and I. Postlethwaite, Multivariable Feedback Control, Wiley, Chichester, England, 1996

[27] Eiji Yokoyama, Masato Nagasawa, and Tsuyoshi Katayama, A disturbance suppression control system for car-mounted and portable optical disk drives, IEEE Transactions on Consumer Electronics 40 (1994), no. 2, 92-99.

[28] Y. Zhou, M. Steinbuch, M.V.D. Aa, and H. Ladegaard, Anti-shock controller design for optical drives, Control Engineering Practice 12 (2004), 811-817. 